\title{
The change of clinical stage after neoadjuvant chemotherapy in prediction of prognosis for borderline resectable and locally advanced pancreatic cancer
}

\author{
Byung-Chang KIM, Woohyung LEE*
}

Division of Hepatobiliary and Pancreatic Surgery, Department of Surgery, Asan Medical Center, University of Ulsan College of Medicine, Seoul, Korea

Introduction: Response evaluation using computed tomography during neoadjuvant chemotherapy (NACT) is limited due to indistinct finding of fibrotic change around tumor. This study was aimed to investigate predictive role of change in clinical stage based on computed tomography (CT) during NACT n patients with borderline resectable pancreatic cancer (BRPC) and locally advanced pancreatic cancer (LAPC).

Methods: BRPC and LAPC patients with NACT using FOLFIRINOX regimen were included between 2012 and 2019 . The patients were divided into responsive, stationary, and aggravated groups based on the change of clinical stage in pre and post NACT CT images. We compared short and long term outcomes among three groups.

Results: Responsive, stationary, aggravated group were found in 37 (22.1\%), 116 (69.4\%), and 16 (9.5\%) patients, respectively. Of 45 patients in pre-NACT stage III, $9(20 \%)$ patients showed downward stage migration in responsive group and 25 (41.5\%) patients were showed upward stage migration in 60 patients with pre-NACT stage I. Responsive group showed the better 3-year recurrence free survival rate compared with stationary and aggravated groups ( $44.2 \%$ vs. $23.5 \%$ vs. $0 \% ; p=0.001)$. Responsive group showed lower proportion of node metastasis $(p=0.048)$, lymphovascular invasion $(p=0.011)$ and smaller tumor size in pathology $(p=0.007)$ compared with other groups.

Conclusions: Responsive group was associated with better recurrence free survival in FOLFIRINOX based NACT. And it may be helpful to manage the patients postoperatively for finding the characteristics of responsive group during NACT for BRPC and LAPC. 